TITLE:

\title{
Mean-field theory of Archimedean and quasicrystalline tilings
}

$\operatorname{AUTHOR}(\mathrm{S})$ :

Dotera, $\mathrm{T}$

CITATION:

Dotera, T. Mean-field theory of Archimedean and quasicrystalline tilings. Philosophical Magazine 2007, 87(18-21): 3011-3019

ISSUE DATE:

2007-07

URL:

http://hdl.handle.net/2433/50526

RIGHT:

この論文は出版社版でありません。引用の際には出版社版をご確認ご 利用ください。; This is not the published version. Please cite only the published version. 


\section{Mean-Field Theory of Archimedean and Quasicrystalline Tilings}

TOMONARI DOTERA

Kyoto University, Katsura, Kyoto 615-8510, Japan

A simple Landau theory of three-component alloy systems under incompressible condition is investigated, which appears to give regions of the phase diagram in which Archimedean tiling phases are stable in two dimensions. Moreover, we find regions where dodecagonal and decagonal quasicrystals appear to be stable. Alexander-MacTague and Mermin-Troian theories of weak crystallization are revisited.

(Received 31 Aug 2006; in final form 24 Oct 2006)

Keywords: quasicrystals; polymers; phase stability, self-assembly; approximants; Archimedean tilings

\section{Introduction}

Recently, several self-organized Archimedean tiling patterns [1], as shown in figure 1 have been obtained in three-component star polymeric alloy systems [2-12]. The structure is a polygonal cylindrical structure like a honeycomb whose cross section is a two-dimensional polygonal tiling. An Archimedean tiling is a tiling in which only one type of vertices is allowed in each tiling. A set of integers, $\left(n_{1}, n_{2}, n_{3}, \cdots\right)$, denotes a tiling of a vertex type in the way that $n_{1}$-gon, $n_{2}$-gon, $n_{3}$-gon, etc, meet consecutively on each vertex. Superscripts are employed to abbreviate when possible. Three Archimedean tilings denoted as $\left(4.8^{2}\right),\left(6^{3}\right)$, and (4.6.12) belonging to the single junction class are particularly important, because they can be colored by three colors, corresponding to three components $[2,3]$. Furthermore, not only the single junction class is observed [4], but also an indirect Archimedean tiling, $\left(3^{2} .4 .3 .4\right)$, composed of equilateral triangles and squares has been observed: This is the first striking experimental realization of a microphase separated structure with complex molecular environments [11]. In metallurgy, the $\left(3^{2} .4 .3 .4\right)$ net is called the $\sigma$ phase known as one of the Frank-Kasper complex alloy family [13-17]. Since the discovery of quasicrystals [18-21], the significance of the $\sigma$ phase is not only its existence, but also its close 
relationship to dodecagonal quasicrystals (DDQC) [22-25]. Both phases always appear with a slight composition change. Hence, a question arises: Can we find polymeric dodecagonal quasicrystals? Remarkably, the $\left(3^{2} .4 .3 .4\right)$ net and a dodecagonal quasicrystal have been formed in a Monte Carlo simulation of the same polymeric star alloys [26].

Three decades ago, the simplest one-order parameter Landau theory [27] for crystallization was proposed by Alexander and MacTague (AM) [28, 29]. They showed that the bcc structure is stable near the liquid-solid transition temperature. Although the theory is independent of details of the system, e.g. the particular atomic species, atomic interactions, temperature, pressure, etc, it accounts for the fact that a large number of metals crystallizes from melts into bcc structures. Surprisingly, a recent experiment on block copolymers showed that a bcc micelle phase form from a melt as well [30]. This implies that the theory appears to be universal over different length scales, regardless of soft or hard matter. In fact, the essence of the AM theory has been adopted by the weak segregation theory of microphase separation of AB block copolymer melts by Leibler, which has succeeded in providing a qualitative understanding of the phase diagram of AB block copolymers [31, 32]. After the discovery of quasicrystals, the AM theory has been extended to two- or multi-order parameter systems by Mermin and Troian (MT) to understand the stability of quasicrystalline phases [33]. Afterwards both AM and MT theories have been further extended to study the stability of dodecagonal quasicrystals and Faraday waves [34-36]. Is the MT-type theory applicable to the multi-component star polymeric alloy system?

In this paper, we first show that the single junction class of the Archimedean tiling patterns of star polymers can be derived naturally from a MT type mean-field theory. Secondly, we present phase diagrams in which complex phases such as $\left(3^{2} .4 .3 .4\right)$, dodecagonal and decagonal phases are stable. Here, we restrict our survey to two-dimensional tiling structures. Therefore, we have ignored lamellae in lamellar-type two-dimensional phase and three-dimensional phases [2].

\section{Two-order parameter theory for $\mathrm{ABC}$ star alloys}

By assuming the incompressibility condition for the densities of star $\mathrm{ABC}$ block copolymer melts, the free energy of three-component alloy systems can be expressed by two order parameters. Since, the inherent three-component nature has not 
been considered in the MT theory, we therefore reconsider the MT theory to construct our minimal model of three-component alloys. In figure 1, a key feature is that three species should meet at vertices and behave like one ingredient, which is realized in the $\mathrm{ABC}$ star polymer system.

According to the AM theory, the third-order term in the free energy gives rise to crystal formation, because the third-order term is associated with three-body interactions that govern how three particles construct triangle in a crystal, whereas the quadratic terms relate to just two-body interactions to determine distances between two particles and to how many atoms exist with respect to a center particle. To explore crystalline or quasicrystalline structures, the relation between three particles is crucially important as compared with liquids and glasses. In the Fourier expansion of the density, sets of $\mathbf{q}$ vectors forming triads contribute to the third-order term. When the magnitude of the reciprocal lattice vectors is restricted to the maximum peak $\mathbf{q}$ of the static structure function, $S(\mathbf{q})$, just above the liquid-solid transition, the problem is reduced to evaluate the number of equilateral triangles in the reciprocal space. The result is a set of vectors consisting of edges of an octahedron leading to the bcc structure in the real space. Similarly, in the two-component theory, the key to determine structures is to construct triangles composed of $\mathbf{q}$ and $\mathbf{k}$ corresponding to two order parameters.

We define the meaningful densities of three components, $\Phi_{\alpha}(\alpha=\mathrm{A}, \mathrm{B}$, or $\mathrm{C})$, as deviations from uniform values. Under the incompressibility condition, $\Phi_{\mathrm{A}}+\Phi_{\mathrm{B}}+\Phi_{\mathrm{C}}=0$, we can describe these densities by using two order parameters, $\Psi$ and $\Phi$ defined as $\Psi \equiv \Phi_{\mathrm{A}}+\Phi_{\mathrm{B}}=-\Phi_{\mathrm{C}}$ and $\Phi \equiv \Phi_{\mathrm{A}}-\Phi_{\mathrm{B}}$. Below we consider the third-order terms of a Landau free energy of the form $-\Phi_{A} \Phi_{B} \Phi_{C}$ and $-\Phi_{C}{ }^{2} \Phi$. The former term represents the effect of three-body attractive interactions such that three components, $\mathrm{A}, \mathrm{B}$, and $\mathrm{C}$ should meet, i.e. $-4 \Phi_{\mathrm{A}} \Phi_{\mathrm{B}} \Phi_{\mathrm{C}}=\Psi^{3}-\Psi \Phi^{2}$. Usually, the third-order term, $\Psi^{3}$, in the AM theory is negative, however, in our definition we find a plus sign, which is very important to determine the phases of density waves. The latter term, $-\Psi^{2} \Phi$, implies that $\Psi$ or $\mathrm{C}$ dominates and induces the whole transition, whereas the $-\Psi \Phi^{2}$ term in turn implies that the phase separation of A and B induces the whole transition. In principle, stepwise transitions of two order parameters can occur; however, these mixed terms reduce this possibility. Since our focus in this paper is the C-dominated transition, the usual AM theory term, $-\Phi^{3}$, is neglected from the starting point as is in the MT theory. 
Now the form of the generic free energy, $f$, describing Archimedean tiling phases and quasicrystals to examine is

$$
f=\frac{1}{V} \int\left[t \Phi^{2}+\Phi^{4}+\tau \Psi^{2}+\Psi^{4}+g_{0} \Psi^{3}-g_{1} \Phi \Psi^{2}-g_{2} \Phi^{2} \Psi\right] d \mathbf{r},
$$

where $t$ and $\tau$ are parameters corresponding to the temperature and each $g_{i}$ is a positive parameter. Two fourth-order term constants are absorbed into the rescaling of $\Phi$ and $\Psi$. The temperature-like parameters, $t$ and $\tau$, control the strength of transitions of $\Phi$ and $\Psi$, respectively. A point $t=0$ (or $\tau=0$ ) is the second-order transition point of a so-called $\Phi^{4}$ model without third-order terms. The third-order terms determine the relation between the sets of reciprocal lattice vectors forming equilateral or isosceles triangles. Before closing this section, we should mention previous studies. When $g_{2}=0$ and the $\Phi^{4}$ term is omitted, the model is the simplest model up to third-order terms, which is nothing but the MT theory. As another extension of the MT theory, a similar idea has been devised by Müller [35] using only one coupled term.

\section{Free Energy Forms of Several Phases}

Below we consider $\mathrm{C}$ dominated transitions; we thus first set up reciprocal lattice vectors $\{\mathbf{k}\}$ for $\Psi(\mathbf{k})$. In figure 2 a-e, the sets of reciprocal lattice vectors $\{\mathbf{k}\}$ (solid line) are illustrated for several phases. By making triads of vectors consisting of $\mathbf{k}$ and $\mathbf{q}$ (broken line) we can construct $\{\mathbf{q}\}$ for $\Phi(\mathbf{q})$. The possible length ratio of vectors can be $1, \sqrt{2}, \sqrt{3}$, or $(\sqrt{5}+1) / 2$. At this stage the $\left(3^{2} .4 .3 .4\right)$ net with vectors $\{\mathbf{k}\}$ having different wavelengths should be out of consideration like the fcc lattice, which has not been considered in the original AM theory. With a sacrifice of simplicity we will include $\left(3^{2} .4 .3 .4\right)$ in the following way [29].

Let $A$ be the lattice constant of the $\left(3^{2} .4 .3 .4\right)$ Bravais lattice spanned by $(A, 0)$ and $(0, A)$. The $\left(3^{2} .4 .3 .4\right)$ crystal structure composed of vertices of squares and equilateral triangles is represented by four-point basis: $(a+c, b+c),(b+c,-a+c),(-a+c$, $-b+c),(-b+c, a+c)$, where $a=(3-\sqrt{3}) A / 4, \quad b=(\sqrt{3}-1) A / 4$ and $c=A / 2$. The reciprocal lattice vector is represented by $\mathbf{G}=m \mathbf{G}_{1}+n \mathbf{G}_{2}$, where $\mathbf{G}_{1}=(2 \pi / A, 0)$, $\mathbf{G}_{2}=(0,2 \pi / A)$. In terms of these basis, the prominent peaks of the structure factor for $\mathrm{C}$ components is given by $\left\{\mathbf{k}_{i}\right\}=\{( \pm 2,0),(0, \pm 2),( \pm 2, \pm 1),( \pm 1, \pm 2)\}$ (figure $\left.2 \mathrm{c}\right)$. The 
subscript $i$ is assigned counterclockwise. The magnitude of vectors for $\mathbf{k}$ is $\sqrt{4}$ or $\sqrt{5}$. Taking this difference into account, we exert a penalty of increase in the coefficients of the second-order terms: $\tau^{\prime}=\tau+c_{\psi}$ as an approximation.

The ( $\left.3^{2} .4 .3 .4\right)$ tiling is composed of squares and equilateral triangle leading to two kinds of local arrangement of $\mathrm{ABC}$ component (figure 1). In addition, both experimentally and numerically this structure is observed in a composition range between $\left(4.8^{2}\right)$, and $(4.6 .12)[3,12]$. Therefore, we adopt two ways to construct $\Phi$. The reciprocal lattice vector for $\phi_{1}$ is the sum of two vectors: $\left\{\mathbf{p}_{i+1}\right\}=\left\{\mathbf{k}_{i}+\mathbf{k}_{i+3}\right\}$ (figure 2(f)), and that for $\phi_{2}$ is the sum $\left\{\mathbf{q}_{i+1}\right\}=\left\{\mathbf{k}_{i}+\mathbf{k}_{i+2}\right\}$ (figure 2h). The magnitude of the vectors is $\sqrt{8}$ or $\sqrt{10}$ for $\mathbf{p}$, and it is $\sqrt{13}$ or $\sqrt{16}$ for $\mathbf{q}$. Taking these differences into account, we exert penalties of increase in the coefficients of the second-order terms: $t^{\prime}=t+c_{\phi}$.

In the case of DDQC, the reciprocal lattice vectors for $\Psi$ are shown in figure 2(d). Since the DDQC is akin to $\left(3^{2} .4 .3 .4\right)$, we employ $\left\{\mathbf{p}_{i+1}\right\}=\left\{\mathbf{k}_{i}+\mathbf{k}_{i+3}\right\}$ and $\left\{\mathbf{q}_{i+1}\right\}=\left\{\mathbf{k}_{i}+\mathbf{k}_{i+2}\right\}$, and the difference between $\mathbf{p}$ and $\mathbf{q}$ is taken into consideration as $t^{\prime}=t+c_{\phi}$, in the same way as $\left(3^{2} \cdot 4.3 .4\right)$.

We can define amplitudes and phases of the Fourier transformation of order parameters: $\Phi\left(\mathbf{q}_{i}\right)=\phi e^{i \alpha_{i}}, \Psi\left(\mathbf{k}_{j}\right)=\psi e^{i \beta_{j}}$. By minimizing free energies with respect to the amplitudes and phases, phases are completely determined except for the phonon and phason degrees of freedom [37]. Attention should be paid to the problem of phases for $\left(6^{3}\right)$, (4.6.12), ( $\left.3^{2} .4 .3 .4\right)$, DDQC and DQC with mutual dependence among the reciprocal vectors.

Here, we explicitly present free energy forms worked out for two types of tetragonal $\left(4.8^{2}\right)(\mathrm{T} 1, \mathrm{~T} 2)$, hexagonal $\left(6^{3}\right)(\mathrm{H} 1)$, hexagonal (4.6.12) (H2), tetragonal $\left(3^{2} .4 .3 .4\right)(\mathrm{T} 3)$, dodecagonal (DD), decagonal (D) and octagonal (O) quasicrystals as follows:

$$
\begin{aligned}
& f_{\mathrm{T} 1}=t \phi^{2}+\frac{9}{4} \phi^{4}+\tau \psi^{2}+\frac{9}{4} \psi^{4}-g_{2} \psi \phi^{2}, \\
& f_{\mathrm{T} 2}=t \phi^{2}+\frac{9}{4} \phi^{4}+\tau \psi^{2}+\frac{9}{4} \psi^{4}-g_{1} \psi^{2} \phi \\
& f_{\mathrm{H} 1}=t \phi^{2}+\frac{5}{2} \phi^{4}+\tau \psi^{2}+\frac{5}{2} \psi^{4}-g_{0} \sqrt{\frac{2}{3}} \psi^{3}-g_{2} \sqrt{\frac{2}{3}} \psi \phi^{2},
\end{aligned}
$$




$$
\begin{aligned}
& f_{\mathrm{H} 2}=t \phi^{2}+\frac{5}{2} \phi^{4}+\tau \psi^{2}+\frac{5}{2} \psi^{4}-g_{0} \sqrt{\frac{2}{3}} \psi^{3}-g_{1} \sqrt{\frac{2}{3}} \psi^{2} \phi \\
& f_{\mathrm{T} 3}=t^{\prime}\left(\phi_{1}{ }^{2}+\phi_{2}{ }^{2}\right)+\frac{37}{12} \phi_{1}^{4}+\frac{11}{4} \phi_{2}{ }^{4}+\frac{17}{18} \phi_{1}{ }^{2} \phi_{2}{ }^{2}-\frac{1}{2} \phi_{1}^{3} \phi_{2}+\tau^{\prime} \psi^{2}+\frac{29}{12} \psi^{4} \\
& -g_{0} \sqrt{\frac{1}{3}} \psi^{3}-g_{1} \sqrt{\frac{1}{3}}\left[\psi^{2} \phi_{1}+\frac{5}{6} \psi^{2} \phi_{2}\right]-g_{2} \sqrt{\frac{1}{3}}\left[\frac{2}{3} \psi \phi_{2}{ }^{2}+\frac{1}{3} \psi \phi \phi_{2}\right] \\
& f_{\mathrm{DD}}=t^{\prime}\left(\phi_{1}{ }^{2}+\phi_{2}{ }^{2}\right)+\frac{11}{4}\left(\phi_{1}{ }^{4}+\phi_{2}{ }^{4}\right)+\frac{4}{3} \phi_{1}{ }^{2} \phi_{2}{ }^{2}+\tau \psi^{2} \\
& +\frac{11}{4} \psi^{4}-g_{0} \sqrt{\frac{1}{3}} \psi^{3}-g_{1} \sqrt{\frac{1}{3}}\left[\psi^{2} \phi_{1}+\psi^{2} \phi_{2}\right] \\
& f_{\mathrm{D}}=t \phi^{2}+\frac{27}{10} \phi^{4}+\tau \psi^{2}+\frac{27}{10} \psi^{4}-g_{1} \frac{2}{\sqrt{10}} \psi^{2} \phi-g_{2} \frac{2}{\sqrt{10}} \psi \phi^{2}, \\
& f_{\mathrm{O}}=t \phi^{2}+\frac{21}{8} \phi^{4}+\tau \psi^{2}+\frac{21}{8} \psi^{4}-g_{1 \mathrm{or} 2} \frac{1}{\sqrt{2}} \psi^{2} \phi .
\end{aligned}
$$

The difference between two $\left(4.8^{2}\right)$ phases is that the length ratio of $\mathbf{k}$ and $\mathbf{q}$ is the inverse, namely $\mathbf{q} / \mathbf{k}=1 / \sqrt{2}$ or $\sqrt{2}$, for $\mathrm{T} 1$ or $\mathrm{T} 2$, respectively. It is generally stated that the more the number of reciprocal vectors is, the more the fourth-order terms. Hence one can deduce that the $\left(4.8^{2}\right)$ phases are low temperature phases, since the fourth-order terms are the smallest among models considered here. Moreover, it is easy to prove analytically that the free energy of the octagonal quasicrystal cannot be lower than that of the $\left(4.8^{2}\right)$ phases over the entire $t-\tau$ range under the assumption of one wavelength for each order parameter. Two $\left(4.8^{2}\right)$ phases are $t-\tau$ (or $\phi-\psi$ ) symmetric, but $\left(6^{3}\right)$ and (4.6.12) are asymmetric due to the $\psi^{3}$ term. As seen in (6) and (7), the dodecagonal quasicrystal and $\left(3^{2} .4 .3 .4\right)$ is very similar in the reciprocal space as well. However, there are subtle differences that stem from nontrivial combination of the $\left(3^{2} .4 .3 .4\right)$ crystalline vectors. Since it is difficult to express free energy minima explicitly as functions of $\tau$ and $t$, in the next section we numerically minimize the free energy forms.

\section{Numerical Results}

The strength of $g_{i}$ coefficients of the third-order terms and two parameters ( $\tau$ and $t$ ) controls the phase behavior. First, it is easy to obtain the $\left(6^{3}\right)$ phase region when $g_{0}$ 
and $g_{2}$ are large enough. This hexagonal symmetry uses the $g_{0}\left(\Psi^{3}\right)$ term, which the original AM theory contains. Striking results originate from the $g_{1}$ and $g_{2}$ terms, demonstrated in figure 3: two phase-diagrams in the $t-\tau$ plane with (a) $g_{0}=0.8$, $g_{1}=2.2, g_{2}=0.2$, and (b) $g_{0}=0.2, g_{1}=2.2, g_{2}=2.2$. Both take values of $c_{\phi}=0.1$, $c_{\psi}=0.2$.

In figure $3 \mathrm{a}$, we find phase regions where $\left(3^{2} .4 .3 .4\right)$ (T3) and dodecagonal (DD) phases are stable in addition to $\left(4.8^{2}\right)$ (T2) and (4.6.12) (H2). The dodecagonal quasicrystal appears on the high temperature side of a wide $\left(3^{2} .4 .3 .4\right)$ phase region. It is reasonable because the $\left(3^{2} .4 .3 .4\right)$ phase is the approximant of the DDQC and the symmetry of the reciprocal vectors for $\psi$ is broken with taking two types of vectors. We note that the additional constant $c_{\psi}$ controls the existence of the DDQC. If $c_{\psi}=0$, the DDQC vanishes in this case. The (4.6.12) phase appears in a region where $\Psi$ dominates over $\Phi$. In other words, the formation of the hexagonal lattice of $C$ component can only cause the whole transition. The $\left(4.8^{2}\right)$ phases should be basically low temperature ones, since the fourth-order terms are small. It is noted that the existence of T1 and T2 near $t=0$ may be an artefact due to the crude approximation by using $c_{\phi}=0.1$.

In figure $3 b$, near the order-disordered phase line, we find a small phase region of the decagonal phase between two types of $\left(4.8^{2}\right)$. A remarkable geometric property should be noticed: both $\psi^{2} \phi$ and $\psi \phi^{2}$ terms exist with respect to the same sets of $\mathbf{k}$ and $\mathbf{q}$ due to the golden triangles (figure $2 \mathrm{i}$ and $\mathrm{j}$ ). It turns out that the decagonal phase region has been obtained near the order-disordered line by taking values of $g_{1}$ and $g_{2}$ the same with keeping $g_{0}$ small.

Finally, in figure 4 we compare a picture of $\Phi_{\mathrm{C}}=-\Psi$ for the dodecagonal quasicrystal with that obtained in the Monte Carlo simulation of $\mathrm{ABC}$ star polymers [26]. The agreement is quite well. The comparison between density waves and a TEM picture for $\left(3^{2} .4 .3 .4\right)$ has been elucidated in Takano et al. [11].

\section{Conclusion}

We have shown that the free energy including third-order terms naturally favours the Archimedean tiling structures, $\left(4.8^{2}\right),\left(6^{3}\right)$, and (4.6.12) belonging to the single junction class. This result is consistent first with the real space geometric analysis [2] and second with the fact that they are easily found in experiments and in MC simulations. It is 
natural that AM and MT theories of weak crystallization is applicable to the star polymers as well, because the essence of the theories is reduced to the combinatorial problem of vectors in the reciprocal space. Moreover, through the geometric interpretations of the third-order terms, we can gain a phenomenological understanding of the phase behavior.

By using only one wavelength for each order parameter, the decagonal phase is stable because of the striking self-similar property of a regular pentagon, whereas the octagonal phase is unstable. It is inevitable that two wavelengths should be used for $\Psi$, as in the case of the FCC lattice. A prescription with a certain loss of simplicity has been used in the case of $\left(3^{2} .4 .3 .4\right)$. In this respect, it is concluded that the dodecagonal quasicrystal phase is more favourable near the melting transition. To stabilize $\left(3^{2} .4 .3 .4\right)$ and DDQC, we believe that the choice of two wavelengths for $\Phi$ is natural; however, we are not certain that the approximation in $t$ adopted here is validated.

\section{References}

[1] B. Grünbaum and G. C. Shephard, Tilings and Patterns (Freeman, NewYork, 1986).

[2] T. Gemma, A. Hatano, and T. Dotera, Macromolecules 35 3225(2002).

[3] K. Ueda, T. Dotera, and T. Gemma, to be published in Phys. Rev. B.

[4] A. Takano, et al., Macromolecules 379941 (2004).

[5] T. Dotera and A. Hatano, J. Chem. Phys. 1058413 (1996).

[6] S. Sioula, N. Hadjichristidis, and E. L. Thomas, Macromolecules 315272 (1998).

[7] S. Sioula, N. Hadjichristidis, and E. L. Thomas, Macromolecules. 318429 (1998).

[7] Y. Bohbot-Raviv and Z.-G. Wang, Phys. Rev. Lett. 853428 (2000).

[8] H. Hückstädt, A. Göpfert, V. Abetz, Macromol. Chem. Phys. 201 296(2000).

[9] K. Yamauchi, et al., Macromolecules 366962 (2003).

[10] A. Takano, et al., J. Polym. Sci. Part B: Polym. Phys. 432427 (2005).

[11] K. Hayashida, et al., Macromolecules 394869 (2006).

[12] F. C. Frank and J. S. Kasper, Acta. Cryst. 12483 (1959).

[13] G. Bergman and D. P. Shoemaker Acta Cryst. 7857 (1954).

[14] A. C. Lawson, C. E. Olsen, J. W. Richardson Jr, M. H. Mueller and G. H. Lander Acta Cryst. B44 89 (1988).

[15] F. Krumeich, M. Conrad, H.-U. Nissen, and B. Harbrecht, Phil Mag. Lett. 78357 (1998). 
[16] G. Ungar, Y. Liu, X. Zeng, V. Percec, W.-D. Cho, Science, 2991208 (2003).

[17] D. Levine and P. J. Steinhardt, Phys. Rev. Lett. 532477 (1984).

[18] D. Shechtman, I. Blech, D. Gratias, and J. W. Cahn, Phys. Rev. Lett. 531951 (1984).

[19] L. Bendersky, Phys. Rev. Lett. 551461 (1985).

[20] N. Wang, H. Chen, and K. H. Kuo, Phys. Rev. Lett. 591010 (1987).

[21] T. Ishimasa, H. -U. Nissen, and Y. Fukao, Phys. Rev. Lett. 55511 (1985).

[22] H. Chen, D. X. Li, and K. H. Kuo, Phys. Rev. Lett. 601645 (1988).

[23] M. Conrad, F. Krumeich, and B. Harbrecht, Angew. Chem. Int. Ed. 371384 (1998).

[24] X. Zeng, G. Ungar, Y. Liu, V. Percec, A. E. Dulcey and J. K. Hobbs, Nature, 428 157 (2004).

[25] T. Dotera and T. Gemma, Phil. Mag. 861085 (2006).

[26] L. D. Landau, Zh. Eksp. Thor. Fiz. 7, 19627 (1937), in The Collected Papers of L.

D. Landau, edited by D. ter Haar (Gordon and Breach-Pergamon, New York, 1965), p.193.

[27] S. Alexander and J. MacTague, Phys. Rev. Lett. 41702 (1974).

[28] P. M. Chaikin and T. C. Lubensky, Principles of condensed matter physics (Cambridge Univ. Press, Cambridge, 1995).

[29] J. Bang and T. P. Lodge, Phys. Rev. Lett. 93245701 (2004).

[30] L. Leibler, Macromolecules, 131602 (1980).

[31] I. W. Hamley, The Physics of Block Copolymer (Oxford Univ. Press, Oxford, 1998).

[32] N. D. Mermin and S. M. Troian, Phys. Rev. Lett. 541524 (1985).

[33] S. Narasimhan and T.-L. Ho, Phys. Rev. B 37800 (1988).

[34] H. W. Müller, Phys. Rev. E, 491273 (1994).

[35] R. Lifshitz and D. M. Petrich, Phys. Rev. Lett. 791261 (1997).

[36] D. Levine, T. C. Lubensky, S. Ostlund, S. Ramaswarmy, and P. J. Steinhardt, Phy. Rev. Lett. 541520 (1985). 


\section{Figure captions}

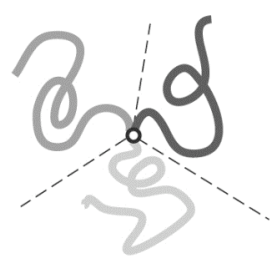

(a)

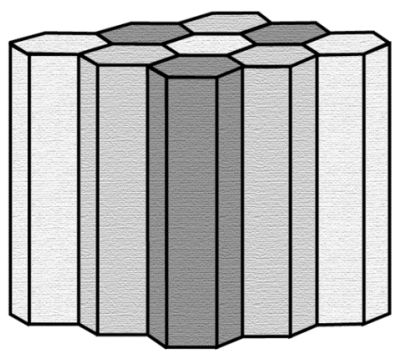

(b)

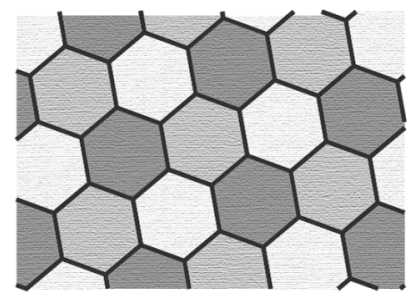

(c) (6.6.6)

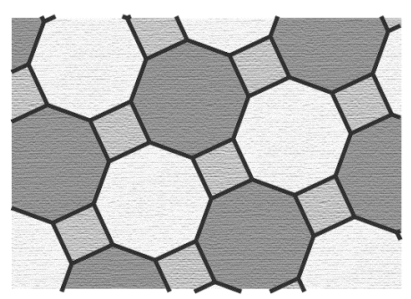

(d)

(4.8.8)

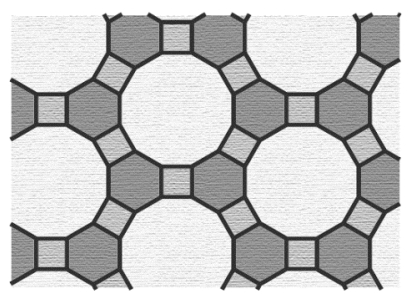

(e) (4.6.12)

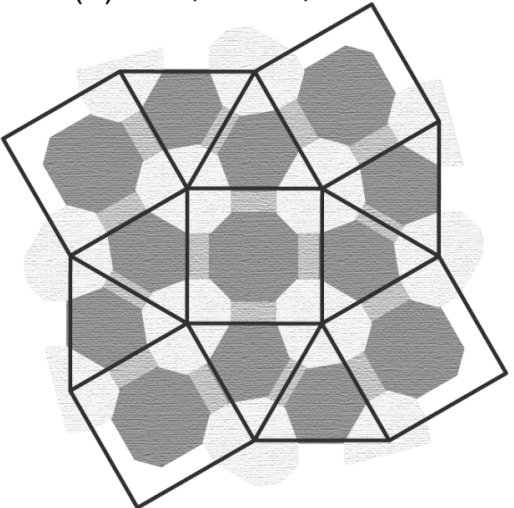

(3.3.4.3.4)

Figure 1. Archimedean tiling phases from ABC star polymers: (a) ABC star block terpolymer; (b) and (c) $\left(6^{3}\right)$, (d) $\left(4.8^{2}\right)$, (e) (4.6.12), (f) $\left(3^{2} .4 .3 .4\right)$ phases. The first three direct patterns constitute the single junction class, whereas the $\left(3^{2} .4 .3 .4\right)$ net gives the skeleton of the real structure.

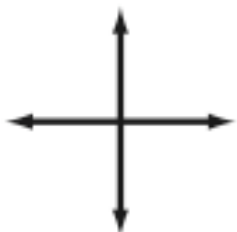

(a)

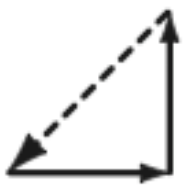

(f)

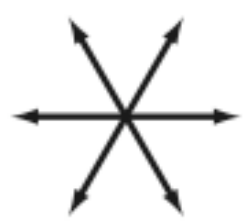

(b)

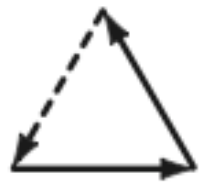

(g)

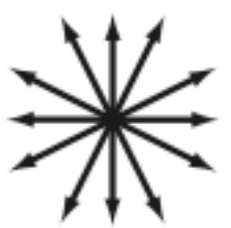

(c)

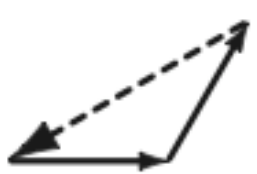

(h)

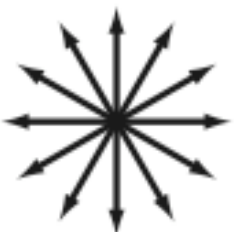

(d)

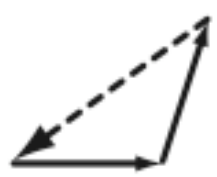

(i)

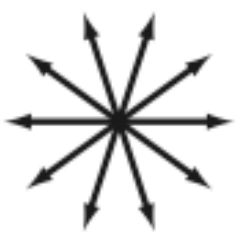

(e)

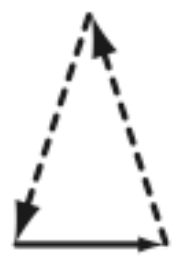

(j)

Figure 2. Sets of reciprocal lattice vectors $\{\mathbf{k}\}$ (solid line) for $\Psi(\mathbf{k})$, and triads of the 
vectors consisting of $\mathbf{k}$ and $\mathbf{q}$ (broken line) for $\Phi(\mathbf{q})$ : (a) $\left(4.8^{2}\right)$, (b) (6 $\left.6^{3}\right)$ and (4.6.12), (c) $\left(3^{2} .4 .3 .4\right)$, (d) dodecagonal quasicrystal (DDQC), (e) decagonal quasicrystal (DQC); the octagonal quasicrystal (OQC) is omitted; a triad (f) is used for $\left(4.8^{2}\right),\left(3^{2} .4 .3 .4\right)$, DDQC, and OQC; $(\mathrm{g})$ is for $\left(6^{3}\right),(\mathrm{h})$ is for (4.6.12), $\left(3^{2} .4 .3 .4\right)$, and DDQC; triads (i) and (j) for DQC are originated from the self-similar property of a regular pentagon.
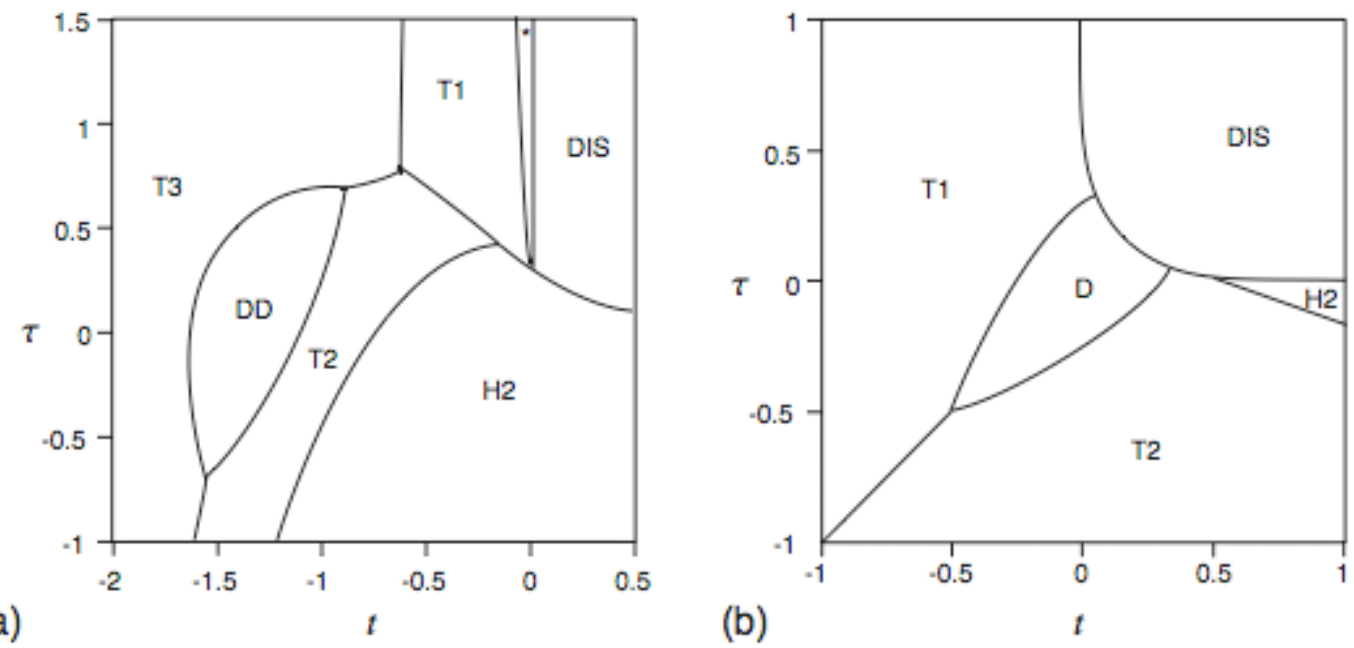

Figure 3. Phase-diagrams in the $t-\tau$ plane: (a) $g_{0}=0.8, g_{1}=2.2, g_{2}=0.2$, and (b) $g_{0}=0.2, g_{1}=2.2, g_{2}=2.2$ with $c_{\phi}=0.1, c_{\psi}=0.2$. T1, T2, T3, H2, denote two types of $\left(4.8^{2}\right),\left(3^{2} .4 .3 .4\right),(4.6 .12)$ Archimedean tiling phases. DD and D represent dodecagonal and decagonal quasicrystals, respectively. DIS implies the disordered phase. In figure (a), a thin region between T1 and DIS with large $\tau$ value represents a tetragonal phase with $\Psi=0$, where a stepwise transition is observed.

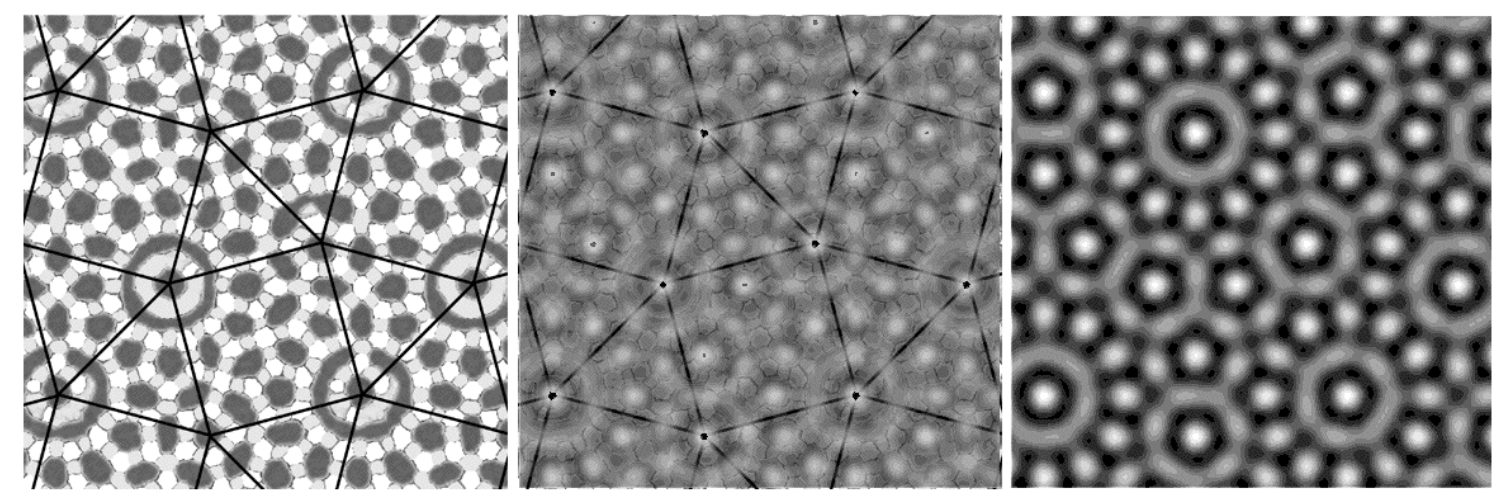

Figure 4. Monte Carlo simulation result (left), the density wave for the $\mathrm{C}$ component obtained from the Landau theory (right), and the superposition (centre). 